\title{
The impact of the coverage of aptamers on a nanoparticle on the binding equilibrium and kinetics between aptamer and protein
}

Xueqian Chen, ${ }^{1,2}$ Fabio Lisi, ,,2 Padmavathy Bakthavathsalam, ${ }^{1,2}$ Guillaume Longatte, ${ }^{1,2}$ Sharmin Hoque, ${ }^{1,2}$ Richard D. Tilley ${ }^{1,3}$ and J. Justin Gooding 1,2*

${ }^{1}$ School of Chemistry, ${ }^{2}$ Australian Centre for NanoMedicine and ARC Centre of Excellence in Convergent Bio-Nano Science and Technology, and ${ }^{3}$ Electron Microscope Unit a Microscopy Australia Node, Mark Wainwright Analytical Centre, University of New South Wales, Sydney, NSW 2052, Australia.

*Corresponding author: J. Justin Gooding

Email: justin.gooding@unsw.edu.au

Characterisations of the AuNPs and AuNPs-aptamer conjugate .....................................2

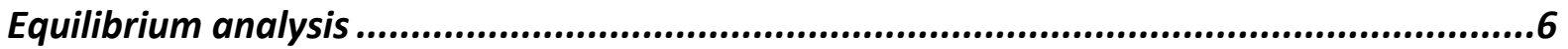

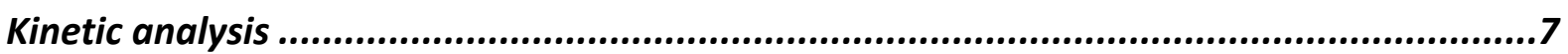

Analytical solution of a reversible second-order rate equation ................................................ 


\section{Characterisations of the AuNPs and AuNPs-aptamer conjugate}
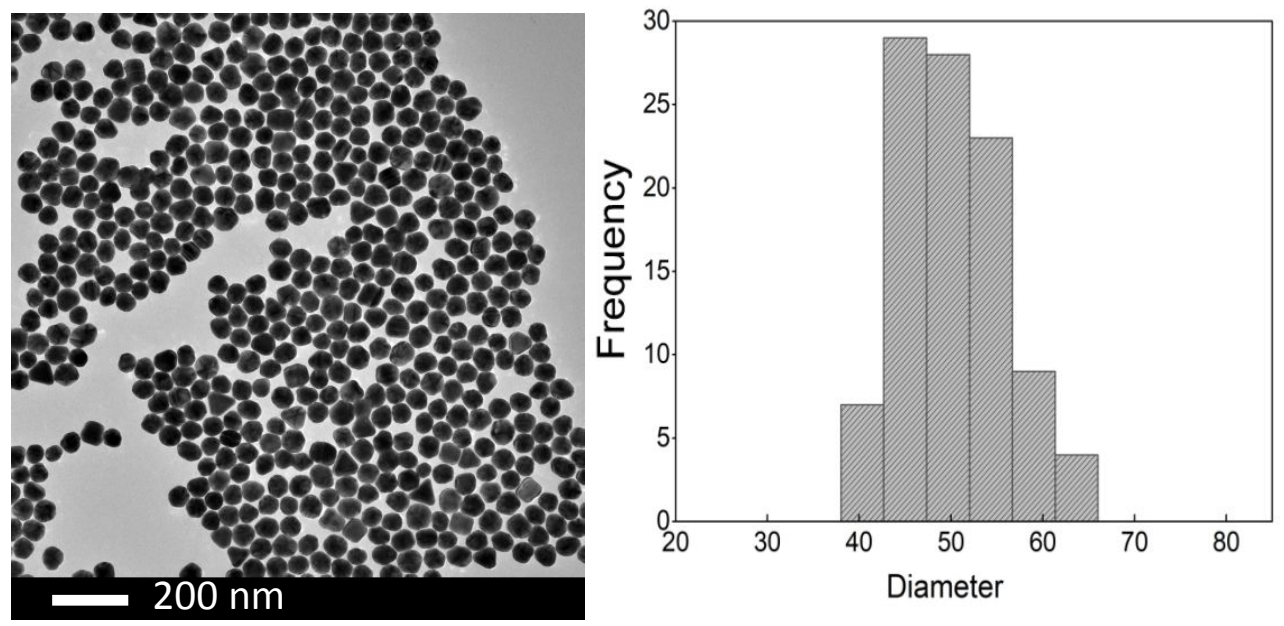

Figure s1. (Left) TEM image of the AuNPs. (Right) Histogram of the particles size. The average particle size is $49 \pm 7 \mathrm{~nm}$.

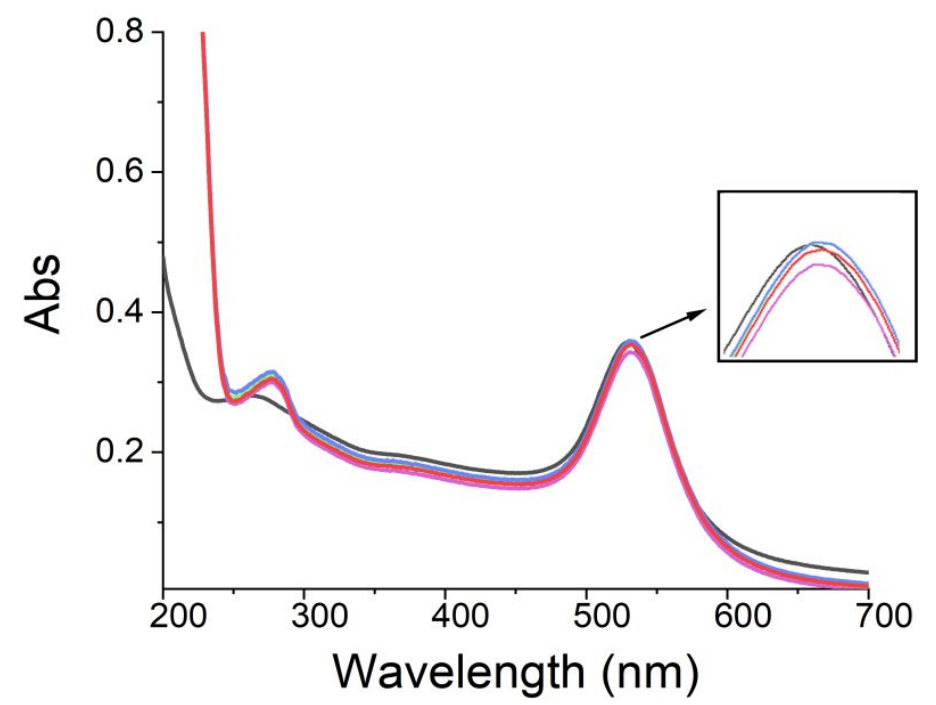

Figure S2 UV-Vis spectra of AuNPs (black line), AuNPsaptamer conjugate with $\mathrm{T}_{10} /$ aptamer $=7: 1$ (purple line), AuNPs-aptamer conjugate with $\mathrm{T}_{10} /$ aptamer $=3: 1$ (green line), AuNPs-aptamer conjugate with $\mathrm{T}_{10} /$ aptamer $=1: 1$ (blue line), and AuNPs-aptamer conjugate with only aptamer (red line). The position of the plasmon resonance peak is $531 \mathrm{~nm}$ for AuNPs and $533 \mathrm{~nm}$ for AuNPs-aptamer conjugate. 


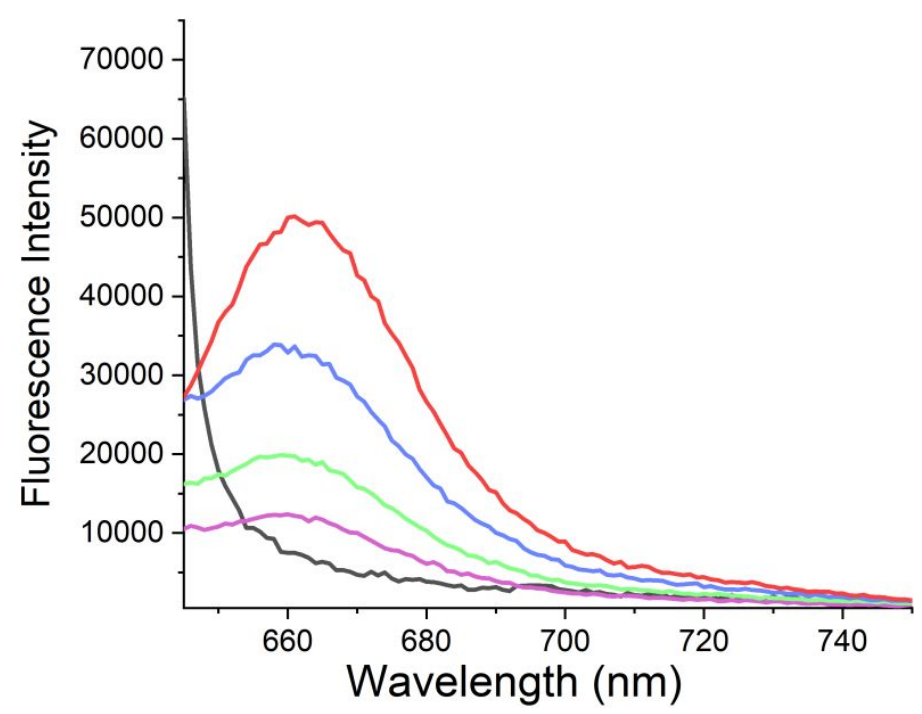

Figure s3 Fluorescence spectra of AuNPs (black line), AuNPs-aptamer conjugate with $\mathrm{T}_{10} /$ aptamer $=7: 1$ (purple line), AuNPs-aptamer conjugate with $\mathrm{T}_{10} /$ aptamer $=3: 1$ (green line), AuNPs-aptamer conjugate with $\mathrm{T}_{10} /$ aptamer = 1:1 (blue line), and AuNPs-aptamer conjugate with only aptamer (red line).

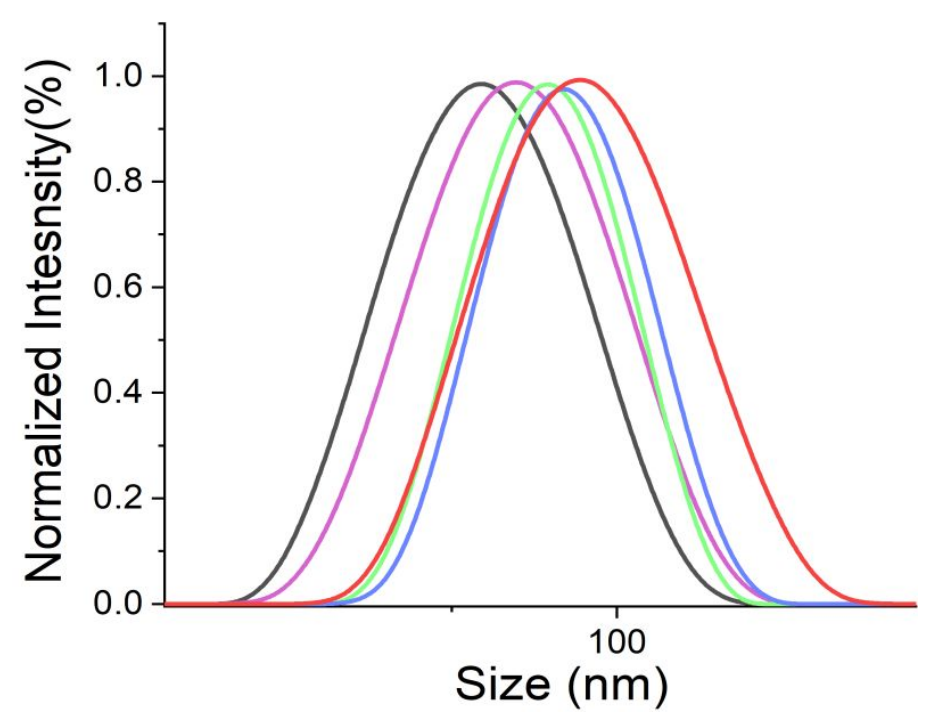

Figure S4 DLS intensity size distribution of AuNPs (black line), AunPs-aptamer conjugate with $\mathrm{T}_{10} /$ aptamer $=7: 1$ (purple line), AuNPs-aptamer conjugate with $\mathrm{T}_{10} /$ aptamer $=$ 3:1 (green line), AuNPs-aptamer conjugate with $\mathrm{T}_{10}$ /aptamer $=1: 1$ (blue line), and AuNPs-aptamer conjugate with only aptamer (red line). 
Table S1. Size, polydispersity Index (PDI) and average area occupied by an aptamer for various surface coverages. The value at saturation (258 aptamers per particle) can be used to find the footprint of the aptamer in its hairpin conformation: $29.2 \mathrm{~nm}^{2}$.

\begin{tabular}{|c|c|c|c|}
\hline & $\begin{array}{l}\text { Intensity } \\
\text { Mean (nm) }\end{array}$ & PDI & $\begin{array}{c}\text { Average area occupied } \\
\text { by an aptamer on the } \\
\text { surface }\left(\mathrm{nm}^{2}\right)\end{array}$ \\
\hline AuNPs & $61.0 \pm 21.28$ & 0.210 & N.A. \\
\hline $\begin{array}{l}\text { Conjugate with } \\
9.6 \text { aptamers per } \\
\text { particle }\end{array}$ & $29.84^{77.96 \pm}$ & 0.125 & 787.4 \\
\hline $\begin{array}{l}\text { Conjugate with } \\
22.3 \text { aptamers per } \\
\text { particle }\end{array}$ & $\begin{array}{l}84.21 \pm \\
27.75\end{array}$ & 0.105 & 338.2 \\
\hline $\begin{array}{l}\text { Conjugate with } \\
46.1 \text { aptamers per } \\
\text { particle }\end{array}$ & $\begin{array}{l}94.58 \pm \\
38.04\end{array}$ & 0.167 & 163.6 \\
\hline $\begin{array}{l}\text { Conjugate with } \\
258 \text { aptamers per } \\
\text { particle }\end{array}$ & $\begin{array}{l}106.7 \pm \\
31.15\end{array}$ & 0.160 & 29.2 \\
\hline
\end{tabular}

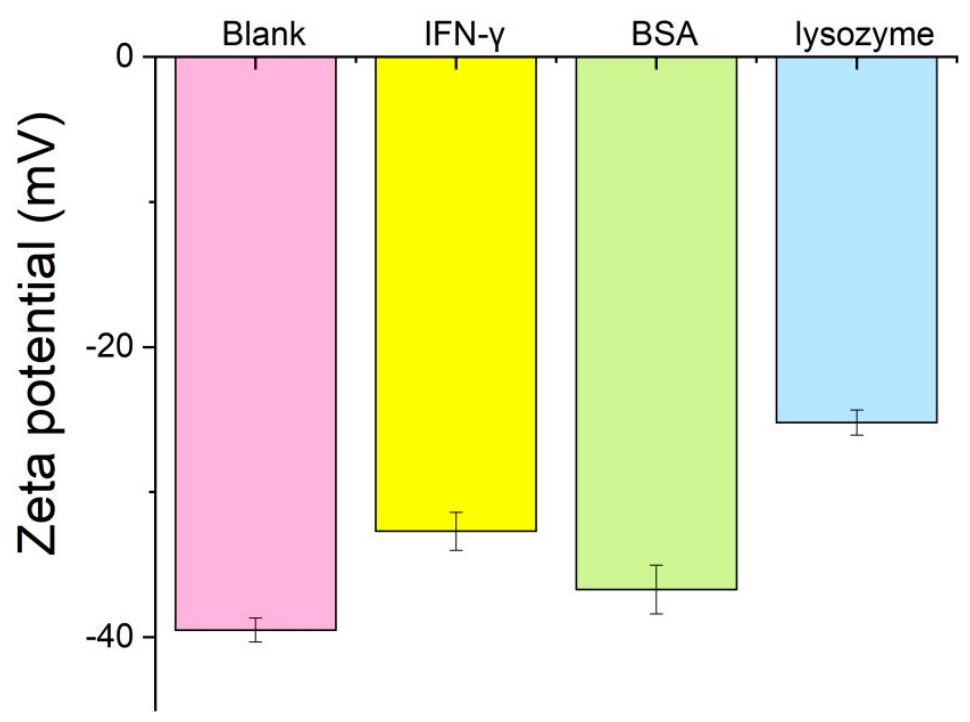

Figure S5 Zeta potential of AuNPs-aptamer conjugate with 22.3 aptamers alone and after incubation with IFN-Y, BSA and lysozyme. 


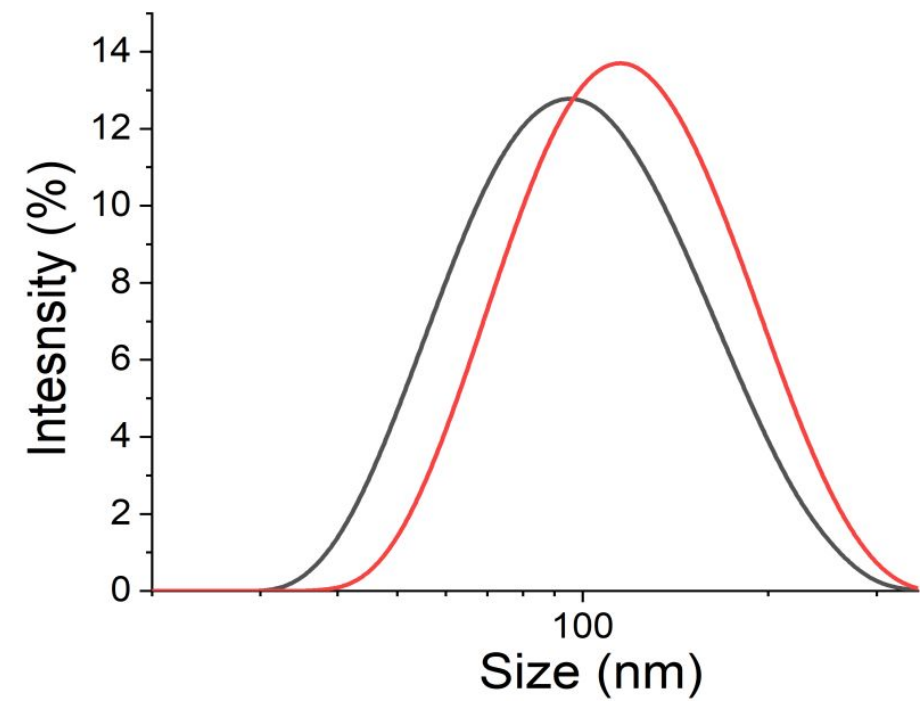

Figure s6. DLS intensity size distribution of AuNPs-aptamer conjugate with 258 aptamers alone and after incubation with 87 $\mathrm{nM}$ of IFN-Y. The size is $107.1 \pm 35.23 \mathrm{~nm}$ for the conjugate without the target and $123 \pm 41.37 \mathrm{~nm}$ when bound to IFN-Y.



Figure S7. Increase of fluorescence intensity of AuNPs-aptamer conjugate with different coverage of aptamer after capturing IFN-Y. 


\section{Equilibrium analysis}

Experimentally, a binding isotherm can be constructed by titrating a fixed concentration of a fluorescently tagged species (AuNPs-aptamer conjugate) with its binding partner (IFNv) that causes the fluorescence to change. It is the concentration of the unlabelled species that is varied because in this way any change in fluorescence is due to the binding. From such data, the equilibrium dissociation constant can be obtained by fitting the data with a suitable model.

The relationship between fluorescence and concentration of nanoparticles at the equilibrium is given again by equation like S2:

$$
F=\alpha_{A}[A]_{e q}+\alpha_{A P}[A P]_{e q}
$$

With $[\mathrm{A}]_{0}=[\mathrm{A}]_{\text {eq }}+[\mathrm{AP}]_{\text {eq }}$. When no IFN-Y has been added, $[A P]_{\text {eq }}=0$, $[A]_{0}=[A]_{e q}$ and the fluorescence is:

$$
F=F_{0}=\alpha_{A}[A]_{0}
$$

At saturation, $100 \%$ of the aptamers have reacted, therefore $[A]_{\text {eq }}$ $=0$ and $[A]_{0}=[A P]_{e q}$ :

$$
F=F_{\text {sat }}=\alpha_{A P}[A P]_{e q}
$$

Therefore, the fluorescence $F$ can be written as:

$$
\begin{gathered}
F=\frac{F_{0}}{[A]_{0}}[A]_{e q}+\frac{F_{\text {sat }}}{[A]_{0}}[A P]_{e q} \\
F=\frac{F_{0}}{[A]_{0}}\left([A]_{0}-[A P]_{e q}\right)+\frac{F_{\text {sat }}}{[A]_{0}}[A P]_{e q} \\
{[A]_{0}\left(F-F_{0}\right)=\left(F_{\text {sat }}-F_{0}\right)[A P]_{e q}} \\
\frac{[A P]_{e q}}{[A]_{0}}=\frac{\left(F-F_{0}\right)}{\left(F_{\text {sat }}-F_{0}\right)}
\end{gathered}
$$

Where $\frac{[A P]_{e q}}{[A]_{0}}$ is the 'bound fraction' $\theta$.

The Hill-Langmuir equation used to fit the data requires the equilibrium concentrations of IFN-Y. Such concentrations were calculated from the mass balance equation of IFN-Y:

$$
\begin{gathered}
C_{I N F \gamma}=[I N F \gamma]_{e q}+[A P]_{e q} \\
{[I N F \gamma]_{e q}=C_{I N F \gamma}-[A P]_{e q}=C_{I N F \gamma}-\left([A]_{0} \frac{\left(F-F_{0}\right)}{\left(F_{s a t}-F_{0}\right)}\right)} \\
\frac{\left(F-F_{0}\right)}{\left(F_{s a t}-F_{0}\right)}=\frac{C_{I N F \gamma}-\left([A]_{0} \frac{\left(F-F_{0}\right)}{\left(F_{s a t}-F_{0}\right)}\right)}{K_{D}^{\prime}+C_{I N F \gamma}-\left([A]_{0} \frac{\left(F-F_{0}\right)}{\left(F_{s a t}-F_{0}\right)}\right)}
\end{gathered}
$$

Equation S10 was used to fit the thermodynamic data. 


\section{Kinetic analysis}

\section{Analytical solution of a reversible second-order rate equation}

The binding between the aptamer-coated nanoparticle and IFN- $\gamma$ is a second-order reversible reaction:

$$
\text { AuNPaptamer }+ \text { IFN-Y } \underset{\mathrm{K}_{\mathrm{D}}}{\rightleftarrows} \text { AuNPaptamer- IFN-Y }
$$

The rate equation is:

$$
-\frac{d[\text { AuNPaptamer }]}{d t}=k_{o n}[\text { AuNPaptamer }][\text { IFN }-\gamma]-k_{o f f}[\text { AuNPaptamer }-I F N-\gamma]
$$

Let us call $\Phi_{\mathrm{A}}$ the fluorescence of aptamers without any IFN-Y at a concentration of 1 unit, and $\Phi_{A P}$ the fluorescence of aptamer bounded to IFN at a concentration equal to 1 unit. The fluorescence of the sample is given by:

$$
F=\Phi_{A}[A]+\Phi_{A P}[A P]
$$

Where $[\mathrm{A}]=[$ AuNPaptamer $]$ and $[\mathrm{AP}]=$ [AuNPaptamer-INFY]. At $t=0$ all the aptamers are without any IFN, as a consequence $[A P]=0$ and $[\mathrm{A}]=[\mathrm{A}]_{0}$. As a consequence:

$$
\begin{aligned}
\Phi_{A}=F_{0} /[A]_{0} \\
\\
F=F_{0} \frac{[A]}{[A]_{0}}+\Phi_{A P}[A P]
\end{aligned}
$$

At equilibrium:

$$
F_{e q}=F_{0} \frac{[A]_{e q}}{[A]_{0}}+\Phi_{A P}[A P]_{e q}
$$

Thus $\Phi_{A P}$ can be written:

$$
\Phi_{A P}=\frac{F_{e q}-F_{0}{ }_{0}^{[A]_{e q}}}{[A P]_{e q}}=\frac{F_{e q}}{[A P]_{e q}}-F_{0} \frac{[A]_{0}-[A P]_{e q}}{[A P]_{e q}[A]_{0}}=\frac{F_{0}}{[A]_{0}}+\frac{F_{e q}-F_{0}}{[A P]_{e q}}
$$

And the fluorescence becomes:

$$
\begin{gathered}
F=F_{0}\left[\frac{[A]}{[A]_{0}}+\frac{F_{0}}{[A]_{0}}[A P]+\frac{F_{e q}-F_{0}}{[A P]_{e q}}[A P]=F_{0} \frac{[A]_{0}-[A P]}{[A]_{0}}+\frac{F_{0}}{[A]_{0}}[A P]+\frac{F_{e q}-F_{0}}{[A P]_{e q}}[A P]\right. \\
F=F_{0}+\frac{F_{e q}-F_{0}}{[A P]_{e q}}[A P]
\end{gathered}
$$

Kinetic law integration:

$$
\begin{gathered}
\frac{d}{d t}[A P]=k_{o n}[A][P]-k_{o f f}[A P]=k_{o n}\left([A]_{0}-[A P]\right)\left([P]_{0}-[A P]\right)-k_{o f f}[A P] \\
\frac{d[A P]}{k_{o n}[A P]^{2}-[A P]\left(k_{o n}\left([A]_{0}+[P]_{0}\right)+k_{o f f}\right)+k_{o n}[A]_{0}[P]_{0}}=d t \\
\frac{d[A P]}{k_{o n}\left([A P]-S_{1}\right)\left([A P]-S_{2}\right)}=d t
\end{gathered}
$$

With $\mathrm{S}_{1}$ and $\mathrm{S}_{2}$ the [AP] values solutions of:

$$
k_{\text {on }}[A P]^{2}-[A P]\left(k_{\text {on }}\left([A]_{0}+[P]_{0}\right)+k_{\text {off }}\right)+k_{\text {on }}[A]_{0}[P]_{0}=0
$$

Let us introduce $\triangle$ such as:

$$
\Delta=\left(k_{\text {on }}\left([A]_{0}+[P]_{0}\right)+k_{\text {off }}\right)^{2}-4{k_{\text {on }}}^{2}[A]_{0}[P]_{0}
$$

It can be seen that $\Delta$ cannot be negative, therefore $S_{1}$ and $S_{2}$ are reals numbers. 


$$
\begin{aligned}
& S_{1}=\frac{k_{o n}\left([A]_{0}+[P]_{0}\right)+k_{o f f}-\sqrt{\Delta}}{2 k_{o n}} \\
& S_{2}=\frac{k_{o n}\left([A]_{0}+[P]_{0}\right)+k_{o f f}+\sqrt{\Delta}}{2 k_{o n}}
\end{aligned}
$$

Because $[A P]_{\text {eq }}$ cannot be above $[A]_{0}$ and $[P]_{0}$, the expression of $[\mathrm{AP}]_{\text {eq }}$ can be written:

$$
[A P]_{e q}=S_{1}=\frac{k_{o n}\left([A]_{0}+[P]_{0}\right)+k_{o f f}-\sqrt{\Delta}}{2 k_{o n}}
$$

As a consequence, we can write:

$$
\frac{d[A P]}{\left([A P]-S_{1}\right)\left([A P]-S_{2}\right)}=\left(\frac{a}{\left([A P]-S_{1}\right)}+\frac{b}{\left([A P]-S_{2}\right)}\right) d[A P]=k_{o n} d t
$$

Let us find $a$ and b:

$$
\frac{d[A P]}{\left([A P]-S_{1}\right)\left([A P]-S_{2}\right)}=\frac{[A P](a+b)-a S_{2}-b S_{1}}{\left([A P]-S_{1}\right)\left([A P]-S_{2}\right)} d[A P]
$$

By identification:

$$
(a+b)=0
$$

Thus:

$$
\begin{gathered}
a=-b \\
-a S_{2}-b S_{1}=b S_{2}-b S_{1}=1 \\
b=\frac{1}{S_{2}-S_{1}} \\
\frac{d[A P]}{\left([A P]-S_{1}\right)\left([A P]-S_{2}\right)}=\left(\frac{\frac{1}{S_{1}-S_{2}}}{\left([A P]-S_{1}\right)}+\frac{\frac{1}{S_{2}-S_{1}}}{\left([A P]-S_{2}\right)}\right) d[A P]=k_{o n} d t \\
\left(\frac{1}{\left([A P]-S_{2}\right)}-\frac{1}{\left([A P]-S_{1}\right)}\right) d[A P]=\left(S_{2}-S_{1}\right) k_{o n} d t
\end{gathered}
$$

Which can be integrate:

$$
\begin{gathered}
\int_{0}^{[A P]}\left(\frac{1}{\left([A P]-S_{2}\right)}-\frac{1}{\left([A P]-S_{1}\right)}\right) d[A P]=\int_{0}^{t}\left(S_{2}-S_{1}\right) k_{o n} d t \\
{\left[\operatorname{Ln}\left(\frac{[A P]-S_{2}}{[A P]-S_{1}}\right)\right]_{0}^{[A P]}=\left[\left(S_{2}-S_{1}\right) k_{o n} t\right]_{0}^{t}=\left(S_{2}-S_{1}\right) k_{o n} t} \\
\operatorname{Ln}\left(\frac{[A P]-S_{2}}{[A P]-S_{1}}\right)-\operatorname{Ln}\left(\frac{S_{2}}{S_{1}}\right)=\left(S_{2}-S_{1}\right) k_{\text {on }} t \\
\frac{[A P]-S_{2}}{[A P]-S_{1}}=\frac{S_{2}}{S_{1}} \operatorname{Exp}^{\left(S_{2}-S_{1}\right) k_{\text {on }} t}=f(t)
\end{gathered}
$$

With $f(t)=\frac{S_{2}}{S_{1}} \operatorname{Exp}^{\left(S_{2}-S_{1}\right) k_{\text {on }} t}$

$$
\begin{gathered}
{[A P]-S_{2}=f(t)\left([A P]-S_{1}\right)} \\
{[A P](1-f(t))=S_{2}-f(t) S_{1}} \\
{[A P]=\frac{S_{2}-f(t) S_{1}}{1-f(t)}=\frac{S_{2}\left(1-\operatorname{Exp}^{\left(S_{2}-S_{1}\right) k_{o n} t}\right)}{1-\frac{S_{2}}{S_{1}} \operatorname{Exp}^{\left(S_{2}-S_{1}\right) k_{o n} t}}=\frac{1-\operatorname{Exp}^{\sqrt{\Delta t}}}{\frac{1}{S_{2}}-\frac{1}{S_{1}} \operatorname{Exp}^{\sqrt{\Delta t}}}}
\end{gathered}
$$

Thus, the fluorescence can be written:

$$
F=F_{0}+\frac{F_{e q}-F_{0}}{[A P]_{e q}}[A P]=F_{0}+\frac{F_{e q}-F_{0} 1-\operatorname{Exp}^{\sqrt{\Delta} t}}{S_{1} \frac{1}{s_{2}}-\frac{1}{s_{1}} \operatorname{Exp}^{\sqrt{\Delta} t}}=F_{0}+\left(F_{e q}-F_{0}\right) \frac{1-\operatorname{Exp}^{\sqrt{\Delta} t}}{\frac{1}{s_{2}}-\operatorname{Exp}^{\sqrt{\Delta} t}}
$$

Equation 542 was then used on Origin Pro to fit the kinetic data. 
The fitting function for the reversible second order rate equation can be downloaded at the

link: https://github.com/Chemistry-fitting-

functions/Chemistry-fitting-functions 
Conjugate with 258 aptamers per particle



Conjugate with 22.3 aptamers per particle

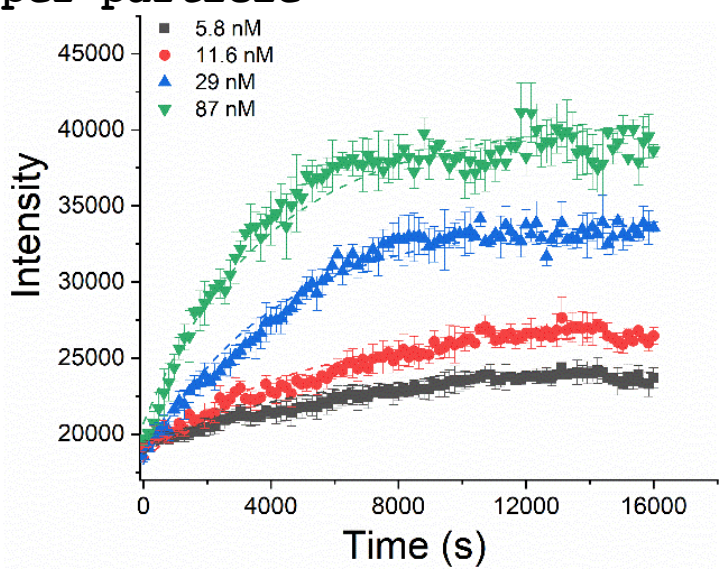

Conjugate with 46.1 aptamers per particle

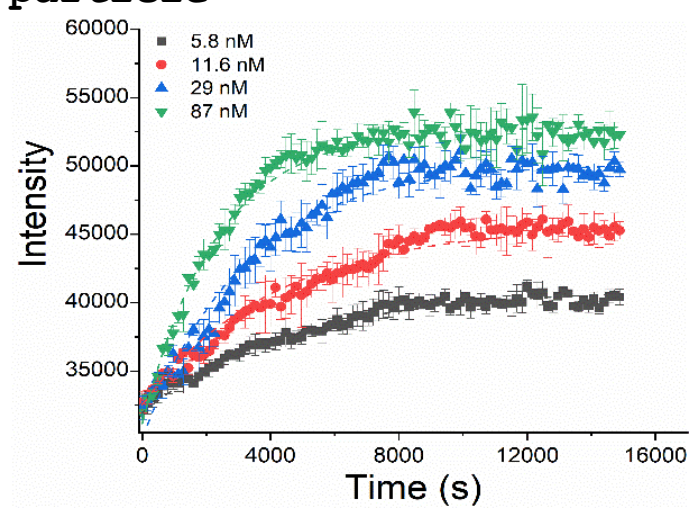

Conjugate with 9.6 aptamers per particle



Figure s8. Kinetic analyses of IFN-Y captured by AuNPs-aptamer conjugate with different surface coverages (from 9.6 to $2.58 \times$ $10^{2}$ aptamers per particle). This set of experiments has been performed with concentration of IFN-Y varying from $5.8 \mathrm{nM}$ to $87 \mathrm{nM}$. The intensity has been fitted to exponential functions and fittings are represented by dash lines. 